\title{
Principles and practice of antibiotic stewardship in the management of diabetic foot infections
}

\author{
Ilker Uçkay ${ }^{\mathrm{a}, \mathrm{b}}$, Martin Berli ${ }^{\mathrm{b}}$, Parham Sendi ${ }^{\mathrm{c}, \mathrm{d}}$, and Benjamin A. Lipsky ${ }^{\mathrm{e}, \mathrm{f}}$
}

\begin{abstract}
Purpose of review
Systemic antibiotic therapy in persons with a diabetic foot infection (DFI) is frequent, increasing the risk of promoting resistance to common pathogens. Applying principles of antibiotic stewardship may help avoid this problem.
\end{abstract}

\begin{abstract}
Recent findings
We performed a systematic review of the literature, especially seeking recently published studies, for data on the role and value of antibiotic stewardship (especially reducing the spectrum and duration of antibiotic therapy) in community and hospital populations of persons with a DFI.
\end{abstract}

\begin{abstract}
Summary
We found very few publications specifically concerning antibiotic stewardship in persons with a DFI. The case-mix of these patients is substantial and infection plays only one part among several chronic problems. As with other types of infections, attempting to prevent infections and avoiding or reducing the spectrum and duration of antibiotic therapy are perhaps the best ways to reduce antibiotic prescribing in the DFI population. The field is complex and necessitates knowledge over the current scientific literature and clinical experience. On a larger scale, clinical pathways, guidelines, and recommendations are additionally supportive.
\end{abstract}

Keywords

antibiotic stewardship, diabetic foot infections, epidemiology, surgery

\section{INTRODUCTION}

Diabetic foot infections (DFIs) are associated with substantial morbidity, frequent and prolonged hospitalizations, a life-long risk for lower extremity amputations, and high financial costs $\left[1-4,5^{*}, 6\right]$. Presented with a patient with a DFI, surgeons and physician want to reduce the risk of poor clinical outcomes. This often leads them to overprescribing antibiotic therapy. This can take the form of prescribing an unnecessarily broad-spectrum regimen (often with combinations of agents), administering parenteral rather than oral therapy, or continuing therapy for a longer duration than necessary [1$\left.4,5^{\mathbf{2}}, 6,7\right]$. Although the clinicians concern about failing to adequately treat infection is understandable, is not only ineffective but associated with risks of adverse events, increased costs and promoting antibiotic resistance. Furthermore, many fail to recognize the importance of other potentially important therapeutic measures, such as pressure offloading or limb revascularization [5"]. Given how common DFIs are, and how difficult they are to treat, they are probably among the most frequent disorders leading to antibiotic overuse worldwide $[8$ ",9].

This overuse of antimicrobial agents, while unlikely to lead to better patient outcomes, does result in both increased financial costs and adverse clinical events $[9,10]$. Looking at the financial side of this issue, annual direct medical costs related for diabetes in the United States alone were estimated at $\$ 176$ billion in 2012 [11]. In a single hospital in Trinidad and Tobago, cost for the care of only 446 patients with DFI was \$14 million US dollars per year [12], which the authors extrapolated to represent

\footnotetext{
${ }^{\mathrm{a} I n f e c t i o l o g y, ~}{ }^{\mathrm{b}}$ Orthopaedic Department, Balgrist University Hospital, Zurich, 'Department of Infectious Diseases and Hospital Epidemiology, ${ }^{\mathrm{d} D e p a r t m e n t}$ of Orthopaedics and Traumatology, University Hospital Basel, University of Basel, Basel, Switzerland, eUniversity of Washington, Seattle, USA and ${ }^{f}$ Green Templeton College, University of Oxford, Oxford, UK

Correspondence to Ilker Uçkay, MD, Balgrist University Hospital, Forchstrasse 340, 8008 Zurich, Switzerland. Tel: +4144386 1111; fax: +41 44386 3709; e-mail: ilker.uckay@balgrist.ch
}

Curr Opin Infect Dis 2019, 32:95-101

DOI:10.1097/QC0.0000000000000530 


\section{KEY POINTS}

- DFls are a major cause of antibiotic misuse and related costs.

- Literature concerning antibiotic stewardship in DFls is sparse.

- Prevention of infections is an important cornerstone of antibiotic stewardship.

- The individual reduction of inappropriate antibiotic use still remains the practice.

- Other approaches, such as clinical pathways with ordersets, are promising.

$0.4 \%$ of the entire gross domestic product of that country. The direct antibiotic-related costs for a DFI may add up to US $\$ 1000$ US dollars in Australia, a resource-rich country [13]. In a recent prospective trial randomizing the use of topical gentamicin sponges (together with systemic antibiotics) for ulcerated DFIs, adverse events occurred at 23\% [14]. Looking at antibiotic-related adverse effects, studies have reported high rates of kidney injuries [15], selection of resistant pathogens such as methicillin-resistant staphylococci or vancomycin-resistant enterococci [16]. The incidence of antibioticresistant pathogens reached $15 \%$ and the rate of transient renal insufficiency reached $30 \%$ in one recent study [15]. Other reported adverse antibiotic-related events include nausea, drug-induced hepatitis, Clostridium difficile colitis [17]), and central-line catheter-related problems from intravenous therapy [15].

In 2016, a joint position paper by the British Society for Antimicrobial Chemotherapy and the European Wound Management Association [18] offered recommendations for wound care, but did not provide in-depth recommendations for antibiotic stewardship in DFIs. In this review, we will summarize the literature related to antibiotic stewardship in DFI. A review of the management of multiresistant pathogens $[19,20]$ and issues related to antibiotic pharmacokinetics or of reimbursement policies $\left[4,5^{\mathbf{*}}, 21\right]$ are beyond the scope of this review.

\section{METHODS}

We conducted a literature search (PubMed and internet) to identify English-language papers up to 23 October 2018 using the MeSH terms: 'infection', 'diabetic foot', 'antibiotic stewardship', 'consumption', 'antibiotic policy', 'pathway', 'guidelines' in different combinations [10]. We were most interested in papers published in the last 2 years.

\section{RESULTS}

We then grouped the retrieved articles of on antibiotic stewardship in DFIs into three major categories: community cases, hospital cases, and prevention of DFIs (Table 1). The majority of identified papers were published between 2 and 10 years ago.

\section{Key antimicrobial stewardship actions}

The most effective measures relating to antibiotic stewardship are: making a correct DFI diagnosis; prescribing an antibiotic regimen with the narrowest effective spectrum [18]; and limiting the duration of antibiotic treatment. Surgically draining and resecting infected and necrotic material can improve the outcome of treatment. Although many clinicians treat DFI for long periods of time, expert guidance suggest that 1 to 2 weeks is sufficient for most soft tissue infections, and 4 to 6 weeks is adequate for osteomyelitis $\left[2,8^{\boldsymbol{*}}, 22,23\right]$. A recent randomized trial comparing 6 versus 12 weeks of antibiotics, without concomitant surgery for diabetic foot osteomyelitis, found no differences in relapse risks [24].

We conducted a retrospective study to investigate whether the route or duration of antibiotic therapy selected was associated with the likelihood of a recurrence of a DFI [8"]. Among 1018 DFI episodes in 482 patients, the median total duration of antibiotic therapy was 20 days. After a median follow-up of 3 years, 251 episodes (24.7\%) recurred, but neither the duration of antibiotic therapy nor the use of parenteral treatment affected the risk of recurrence [ $\left.8^{*}\right]$. Therapy with oral antibiotic agents can be effective [9] in nonbacteremic mild and moderate DFIs $\left[2,8^{\mathbf{*}}, 14,22,23\right]$, which are by far the majority of cases. Reports of oral antibiotic therapy for DFIs, including cases with osteomyelitis, have been published since 1985 [22]. The results of a review of 93 DFI cases published in 2006 strongly supported treatment with oral antibiotic regimens right from the start in cases of diabetic foot osteomyelitis [22]. Data from studies of treatment of other types of osteomyelitis support the effectiveness of oral antibiotic therapy [23]. Unfortunately, there is a persistent reluctance to select oral antibiotic regimens for diabetic foot osteomyelitis, largely based on concern that concomitant arteriopathy may limit delivery to the foot, or the poor bone penetration would hamper clinical efficacy. We found no published data to support these fears. Available retrospective and prospective analyses 
Table 1. Summary of possible measures to promote antibiotic stewardship in diabetic foot infections (authors' selection)

\begin{tabular}{|c|c|c|c|c|}
\hline Setting & Group action & Individual action & Difficulties & Reference \\
\hline \multirow[t]{4}{*}{ Community } & Statewide policies & & No specific governmental initiatives found & - \\
\hline & Treatment guidelines & Less antibiotic use & None & Lipsky [2] \\
\hline & Diabetic foot centers & & No published proof of antibiotic prescribing & - \\
\hline & Outcome benchmarking & & Varied case-mix clouds interpretation & Milne [32] \\
\hline \multirow{13}{*}{$\begin{array}{l}\text { Healthcare } \\
\text { institutions }\end{array}$} & Stewardship programs & Less antibiotic use & Resource-consuming & Lipsky [18] \\
\hline & $\begin{array}{l}\text { Withhold antibiotics } \\
\text { before sampling }\end{array}$ & & Reluctance to withdrawal antibiotics & Al-Mayahi [38] \\
\hline & $\begin{array}{l}\text { Guidelines for } \\
\text { microbiological sampling }\end{array}$ & & None & Sotto $[41]$ \\
\hline & Restriction of selected antibiotics & & Difficult to gain accepted by clinicians & Lipsky [18] \\
\hline & Cycling of antibiotic agents & & No published data for diabetic foot infections & - \\
\hline & Clinical pathway & & Targets mostly amputations and ulcer care & Martinez [39] \\
\hline & $\begin{array}{l}\text { Link doctors/nurses } \\
\text { (dedicated staff) }\end{array}$ & & Not explored in the literature & - \\
\hline & Feedback of results & & Benefit not specifically reported in the literature & Lipsky [18] \\
\hline & Professional wound care & & None & Lipsky [2] \\
\hline & Bacteriophage therapy & & Minimal published evidence of clinical efficacy & Chibber [31] \\
\hline & & $\begin{array}{l}\text { Topical } \\
\text { antiseptics }\end{array}$ & No benefit if combined with systemic antibiotics & Abbas [29] \\
\hline & Infectiology consultant & & Availability of ID consultant & Uçkay [37] \\
\hline & New diagnostic tools & & Unclear influence on antibiotic prescribing & Lavigne [42] \\
\hline \multirow[t]{6}{*}{ Prevention } & Optimal surgical techniques & & Surgeon and institution-dependent & Uçkay [4] \\
\hline & Proper antibiotic prophylaxis & & None & Uçkay [21] \\
\hline & S. aureus decolonization & & $\begin{array}{l}\text { Not specifically analyzed for antibiotic } \\
\text { stewardship }\end{array}$ & - \\
\hline & Antibacterial vaccines & & $\begin{array}{l}\text { Not specific enough for polymicrobial } \\
\text { infections }\end{array}$ & - \\
\hline & Pressure off-loading & & Patient's compliance & Uçkay [3] \\
\hline & Patient education & & Patient's compliance and understanding & Lipsky [2] \\
\hline
\end{tabular}

have demonstrated the noninferiority of oral medication (or rejected the superiority of parenteral dosing) $\left[1-4,5^{\mathbf{*}}, 22\right]$.

\section{Gaps in research}

Data available from published studies and expert recommendations still leave questions about optimal therapeutic approaches, the answer to which might provide methods to reduce unnecessary antibiotic therapy. For examples, results of some as yet unpublished studies suggest we might be able to treat properly selected mild DFIs [2] without topical or systemic antibiotic therapy. Although it was previously assumed that every nonamputated DFI requires treatment with antimicrobials, a recent prospective randomized trial found that mild DFIs could be effectively treated solely with appropriate local wound care [25].

Another example is that many clinicians broaden the spectrum of their empiric antibiotic regimen after a patient has a clinical recurrence of a recently treated DFI. We showed that pathogens causing iterative DFI episodes, in the same anatomical localization, were less antibiotic-resistant than in the previous ones. Indeed, in two-thirds of clinical recurrences the pathogens are different from those that caused the earlier episodes [26]. Thus, these data suggest there is no necessity to broadening the empiric antibiotic spectrum. Similarly, colonization of the patient with healthcare-associated methicillin-resistant S. Staphylococcus aureus (MRSA) rarely requires targeted anti-MRSA therapy $[19,27]$ and obligate anaerobes are often innocent by-standers in polymicrobial soft tissue infections that do not need targeted antibiotic therapy [28].

Treatment with topical antibiotic (or antiseptic) agents often fails to enhance treatment success of DFI, especially when combined with systemic antibiotic therapy [29]. In a pilot study in which we randomized patients with DFI to be treated with a 
gentamicin-collagen sponge as an adjunct to systemic antibiotic therapy versus systemic antibiotic therapy alone, we found no difference in outcomes [14]. In terms of new classes of therapeutic options, we currently lack alternative compounds. Results of studies of treatment with topical antimicrobial peptides (e.g., pexiganan) for DFI were initially promising [30], but more recent studies have yielded disappointing results. Likewise, although early results of therapy with virus bacteriophages appear promising, it may need to be conducted in conjunction with systemic antibiotics [31].

\section{The community setting}

There are administrative approaches that might improve the use of antimicrobials. Governments could establish specialized diabetic foot care centers, which could perhaps reduce inappropriate antibiotic prescribing, compared to current dispensers [12], and provide regular workshops and public educational lectures. To date, however, there is no published evidence that treatment in specialized centers leads to more rational antibiotic therapy. Benchmarking [32] of antimicrobial use and clinical outcomes among centers, and publicly reporting their results, would be challenging and potentially produce erroneous results because of the variations in case-mix of DFI seen in different centers. In contrast, regional or international guidelines, some of which have been amply validated and are updated, are available and published [2,33,34]. These guidelines can help investigators to conduct evidence-based therapies underpinned by antimicrobial stewardship principles; while they may improve treatment outcomes [35].

\section{The hospital setting}

Treatment in healthcare facilities is the best explored setting for investigating antibiotic stewardship [36,37]. This is related to several factors, including a public commitment to improve care and provide transparency; more frequent treatment by multidisciplinary teams; the development and use of clinical pathways; and the presence of academic physicians interesting in investigating their local processes and outcomes [36]. Moreover, healthcare institution more often have ready access to an infectious diseases/clinical microbiology physician familiar (and usually involved) with antimicrobial stewardship programs. Such programs have been shown to help reduce cost-effectively antibiotic consumption [37]. Another method healthcare institutions use to control inappropriate antibiotic use is to mandate constraining the use and duration of empirical preoperative and postoperative antibiotic treatment [38]. Presurgical antibiotic treatments in clinically stable patients with DFI are seldom beneficial and tend to select antibiotic-resistant pathogens. This often results in clinicians administering broad-spectrum antibiotic therapy, sometimes related to the fact that the true pathogens are masked by antibiotic-resistant organisms (which are often only colonizers) growing from specimen cultures. In a review of 2740 orthopedic infections, including DFIs, we found that presurgical antibiotic treatment (including single-dose prophylaxis) was associated with a three-fold higher incidence of antibiotic-resistant nonfermenters and skin commensals, and negative cultures [38].

Instituting clinical pathways has been a frequently used method to improve outcomes, including for DFIs [39]. Although multidisciplinary (or, more accurately, interdisciplinary) teams have clearly been shown to be beneficial, they are hampered by three major problems: it is often difficult to bring the team members together when a patient needs them outside of a fixed meeting time; the number of patients requiring evaluation often exceeds the capacity of fixed meetings; team meetings are time-consuming, usually unremunerated and key members may be absent. Order-sets [36] are another powerful tool to rapidly implement 'bundles' (a package of orders designed to ensure uniform processes) and improve outcomes. Recent published papers have mainly reported on clinical pathways targeted at all foot problems, especially treating or preventing ulcers [40], reducing lower extremity amputations [39] or avoiding or shortening hospital stays [40]. There are few studies primarily designed to improve antibiotic treatment of DFI. Sotto et al. [41] demonstrated that employing expanded guidelines and education led to a significant decrease in the number of bacteria and multidrug-resistant organisms per foot infection sample. In parallel, there was an associated cost savings of $€ 14$ 914 related to a reduced microbiology laboratory workload and another $€ 109305$ because of reduced prescribing of extended-spectrum antibiotics [41].

\section{The role of microbiological diagnostic tests}

New molecular diagnostic methods have revealed the more complex microbiology of DFI than was appreciated with standard culture methods, but it is not yet clear whether this information will contribute to antibiotic stewardship [42], as the clinical importance of the increased number of species identified by these methods remains to be elicited. We also have explored the possible use of antistreptolysin antibodies for the serological identification of 
streptococci (with subsequent reduction of the antibiotic spectrum to penicillins) [43], but it does not appear to be of any practical use in DFI. Pro-calcitonin could be promising, but its benefit in reducing antibiotic consumption in DFI needs to be evaluated more, because several studies deny its diagnostic accuracy in localized orthopedic infections promising without systemic inflammation, including DFI [44].

In addition, there are some rules regarding the handling of already established microbiological tests, which all add to antibiotic stewardship. For instance, bone culture is only needed when osteomyelitis is suspected and infected bone remains in the tissue. In clinically stable patients it is preferable to collect specimens after an antibiotic-free interval of 1 to 2 weeks and via an image-guided technique or during surgical intervention [45]. Cultures of softtissues (even deep specimens close to bone) or sinus tract cultures do not correlate accurately with bone pathogens. Also, clinicians should avoid collecting specimens with swabs, as they are frequently contaminated with skin flora and do not allow for histological examination. Close collaboration between clinicians and microbiology laboratory personnel regarding the clinical disease, location, and type of biopsy specimens, and proper transfer of specimens will produce the most accurate results. These steps may specifically help in interpreting culture results and avoiding unnecessary antimicrobial susceptibility testing for commensal skin flora. Clinician-microbiologist interactions can also optimize weighting the results of present and previous culture results (if available), and in decision-making for appropriate treatment duration.

\section{Antibiotic reduction via prevention and evidence-based surgery}

Prevention of infection is an obvious way to reduce antibiotic consumption. In particular, prolonged or exaggerated preoperative antibiotic prophylaxis is unnecessary for most patients with diabetes who undergo surgery for noninfectious problems such as fractures [46] or correction of anatomical alterations (induced by the neuropathy $\left[1-4,5^{\circ}\right]$ inherent to practically all patients with chronic diabetes). Proper surgical technique and expertise are important in reducing postoperative infections, but assessing these is subjective and difficult [4]. Employing excellent surgical technique, such as maintaining effective hemostasis, gentle tissue handling, removal of devitalized tissue, and dead space eradication, reduces surgical site infections [46]. For established DFIs, in most cases the cavity should be left open for secondary wound healing, as primary wound closure increases the risk of persistent infection. Finally, a longer duration of surgery is a strong predictor of surgical site infections [21]. Optimizing surgical preparation and intraoperative techniques can improve outcomes and can be learned [46].

\section{Promising avenues of research}

Information from recently published literature offers new measures for preventing SSI and reducing inappropriate antibiotic treatment. Although some results appear to be contradictory at the present, they offer possibilities for future research.

\section{Duration of antibiotic treatment for residual stump osteitis after amputation}

In current practice, antibiotic prescribing after complete toe amputation ranges from a few days of oral therapy to several weeks of intravenous therapy. Kowalski et al. [47] demonstrated that patients with a positive culture of the resection margin for residual osteomyelitis had more failures than those without (44 vs. 15\%, despite 2 weeks of antibiotic therapy in both arms). Atway et al. [48] reported a $41 \%$ incidence of positive bone culture margins among 27 trans-osseous amputations, compared to a $23 \%$ following disarticulation. The presence of positive margins was associated with worse outcomes, again despite 25 days of postsurgical antibiotic therapy. More recently, Mijuskovic et al. [49] suggested that the assessment of residual bone infections should perhaps not only rely on cultures, as positive cultures without concomitant histological confirmation might overestimate the true rate of osteomyelitis, presumably related to specimen contamination at the time of surgery. A definite answer to this question certainly will also influence future antibiotic recommendations.

\section{Use or oral $\beta$-lactam antibiotics for diabetic foot osteomyelitis}

Many experts in orthopedic infections avoid ordering oral $\beta$-lactam monotherapy for implant-related orthopedic infections or chronic osteomyelitis, because of their reported limited oral bioavailability and poor bone penetration from in vitro assessments [50]. By analogy, most experts argue against using any oral $\beta$-lactam for DFI, especially in the context of osteitis. However, oral amoxicillin/clavulanic acid is among the most frequently administered $\beta$-lactams for DFI worldwide $\left[8^{*}, 9,40\right]$. Many practice-oriented recommendations [2] mention it as a valuable alternative for mild and moderate DFI. In light of the potential benefit for antibiotic stewardship programs, oral $\beta$-lactams should be further explored. 
Table 2. Ten major rules for antimicrobial stewardship in diabetic foot infection (DFI)

Prevent DFI: Closely monitor diabetic patients at risk for infection and optimize all aspects related to foot care, including appropriate foot ware, nail and skin care and treatment of any wounds.

Diagnosing DFI correctly: Be precise and consistent when diagnosing, and describing the severity of, infection.

Exclude noninfectious causes of foot inflammation: These include trauma, gout/pseudogout, Charcot neuro-osteoarthropathy, fracture, phlebothrombosis, and venous stasis.

Identify causative pathogens: Send samples of tissue (not swabs) and preferably bone (for suspected osteomyelitis). Review results of any previously obtained cultures. Attempt to differentiate pathogens (requiring treatment) from colonizing or contaminating organisms.

Differentiate between soft-tissue and bone infection: This helps in making decisions with respect to medical versus surgical, urgency of, and duration of, treatment.

Ensure specialist consultation for most moderate and all severe infections: Evaluate and individualize treatment options for each patient. In complex cases, involve specialists (or optimally multidisciplinary teams), especially surgeons.

Choose an effective antibiotic regimen with the narrowest spectrum: For empiric, and especially definitive, therapy select an antibiotic regimen based on the likely or proven: causative pathogen(s); their antibiotic susceptibilities; and, evidence of efficacy for DFls. Consider testing in cases of reported 'penicillin allergy'.

Optimize patient-related effectiveness of antibiotic therapy: Evaluate for factors such as adherence to the treatment regimen, impaired gastrointestinal absorption, key comorbidities (obesity, renal failure), and presence of clinically significant peripheral arterial disease in affected limb.

Medical therapy - as long as necessary and as short as possible: For most mild and moderate soft tissue infections 1-2 weeks of therapy is sufficient. For osteomyelitis with residual infected bone, prescribe no more than 6 weeks of antibiotic therapy. Consider shorter treatment durations if infection resolves quickly.

Surgical treatment - can limit need for antibiotic therapy: Draining abscesses and resecting infected bone can limit the duration of antibiotic therapy required.

\section{Screening for Staphylococcus aureus carriage with subsequent decolonization}

$S$. aureus carriage in a patient or treating clinician is not only a nosocomial risk for (staphylococcal) surgical site infections in general surgery, but potentially also a risk for iterative communitary staphylococcal soft-tissue infections [51]. There is still a debate as to whether $S$. aureus in the anterior nares and in a diabetic foot wound of the same patient are concordant [52] or not [53]. As a group, patients with orthopedic conditions appear to benefit from attempts to 'search-and-destroy' $S$. aureus colonization, both in reduction of surgical site infection and in a cost-saving [21]. However, there is as yet no literature that has specifically looked at this issue for DFI surgery.

\section{CONCLUSION}

Available literature concerning antibiotic stewardship specifically designed for DFI patients is unfortunately sparse and further research is greatly needed. Table 2 recapitulates and summarizes our 10 rules of antibiotic stewardship in DFIs. Among all possible approaches, general efforts to prevent DFIs and individualized reduction of unnecessary or inappropriate antibiotic use are probably the best current practices. We need further research for these, and other suggested approaches.

\section{Acknowledgements}

None.

\section{Financial support and sponsorship} None.

\section{Conflicts of interest}

There are no conflicts of interest.

\section{REFERENCES AND RECOMMENDED READING}

Papers of particular interest, published within the annual period of review, have been highlighted as:

- of special interest

- of outstanding interest

1. Uçkay I, Aragón-Sánchez J, Lew D, Lipsky BA. Diabetic foot infections: what have we learned in the last 30 years? Int J Infect Dis 2015; 40:8191.

2. Lipsky BA, Berendt AR, Cornia PB, et al. 2012 Infectious Diseases Society of America (IDSA) clinical practice guideline for the diagnosis and treatment of diabetic foot infections. Clin Infect Dis 2012; 54:132-173.

3. Uçkay I, Gariani K, Pataky Z, Lipsky BA. Diabetic foot infections: state-of-theart. Diabetes Obes Metab 2014; 16:305-316.

4. Uçkay I, Gariani K, Dubois-Ferrière V, et al. Diabetic foot infections: recent literature and cornerstones of management. Curr Opin Infect Dis 2016; 29:145-152.

5. Uçkay I, Jornayvaz FR, Lebowitz $D$, et al. An overview on diabetic foot

- infections, including issues related to associated pain, hyperglycemia and limb ischemia. Curr Pharm Des 2018; 24:1243-1254.

This review resumes the most up-to-date knowledge on the global management of diabetic foot infection in 2017.

6. Tobalem M, Uçkay I. Evolution of a diabetic foot infection. New Engl J Med 2013; 369:2252.

7. Conterno LO, Turchi MD. Antibiotics for treating chronic osteomyelitis in adults. Cochrane Database Syst Rev 2013; 9:CD004439.

8. Gariani K, Lebowitz D, von Dach E, et al. Remission in diabetic foot infections:

- duration of antibiotic therapy and other possible associated factors. Diabetes Obes Metab 2018. [Epub ahead of print]

This retrospective cohort analysis investigates if there is a minimal threshold of antibiotic administrations for various subsets of diabetic foot infections. There is none and the authors discuss the possibilities for antibiotic reduction; basing on their single-center cohort.

9. Lipsky BA. Diabetic foot infections: current treatment and delaying the 'postantibiotic era'. Diabetes Metab Res Rev 2016; 32:246-253. 
10. Davey $P$, Brown $E$, Charani $E$, et al. Interventions to improve antibiotic prescribing practices for hospital inpatients. Cochrane Database Syst Rev 2013; CD003543.

11. Centers for Disease Control and Prevention. 2014 National Diabetes Statistics Report. https://www.cdc.gov/diabetes/pdfs/data/2014-report-estimates-of-diabetes-and-its-burden-in-the-united-states.pdf. [Accessed 29 October 2018]

12. Cawich $S O$, Islam $S$, Hariharan $S$, et al. The economic impact of hospitalization for diabetic foot infections in a Caribbean nation. Perm J 2014; 18:101-104.

13. Commons RJ, Robinson $\mathrm{CH}$, Gawler D, et al. High burden of diabetic foot infections in the top end of Australia: an emerging health crisis (DEFINE study). Diabetes Res Clin Pract 2015; 110:147-157.

14. Uçkay I, Kressmann B, Malacarne S, et al. A randomized, controlled study to investigate the efficacy and safety of a topical gentamicin-collagen sponge in combination with systemic antibiotic therapy in diabetic patients with a moderate or severe foot ulcer infection. BMC Infect Dis 2018; 18:361.

15. Van Asten SAV, Mithani M, Peters EJG, et al. Complications during the treatment of diabetic foot osteomyelitis. Diabetes Res Clin Pract 2018; 135:58-64.

16. Uçkay I, Pires D, Agostinho A, et al. Enterococci in orthopaedic infections: who is at risk getting infected? J Infect 2017; 75:309-314.

17. Schindler M, Bernard L, Belaieff W, et al. Epidemiology of adverse events and Clostridium difficile-associated diarrhea during long-term antibiotic therapy for osteoarticular infections. J Infect 2013; 67:433-438.

18. Lipsky BA, Dryden M, Gottrup F, et al. Antimicrobial stewardship in wound care: a Position Paper from the British Society for Antimicrobial Chemotherapy and European Wound Management Association. J Antimicrob Chemother 2016; 71:3026-3035.

19. Reveles KR, Duhon BM, Moore RJ, et al. Epidemiology of methicillin-resistant Staphylococcus aureus diabetic foot infections in a large academic hospital: implications for antimicrobial stewardship. PLoS One 2016; 11:0161658.

20. Huttner B, Harbarth S, Nathwani D; ESCMID Study Group for Antibiotic Policies (ESGAP). Success stories of implementation of antimicrobial stewardship: a narrative review. Clin Microbiol Infect 2014; 20:954-962.

21. Uçkay I, Hoffmeyer $P$, Lew $D$, Pittet $D$. Preventing surgical site infections. Expert Rev Anti Infect Ther 2010; 8:657-670.

22. Embil JM, Rose G, Trepman E, et al. Oral antimicrobial therapy for diabetic foot osteomyelitis. Foot Ankle Int 2006; 27:771-779.

23. Spellberg B, Lipsky BA. Systemic antibiotic treatment of chronic osteomyelitis in adults. Clin Infect Dis 2012; 54:393-407.

24. Tone A, Nguyen S, Devemy F, et al. Six-week versus twelve-week antibiotic therapy for nonsurgically treated diabetic foot osteomyelitis: a multicenter open-label controlled randomized study. Diabetes Care 2015; 38:302-307.

25. Uçkay I, Kressmann B, Di Tommaso S, et al. A randomized controlled trial of the safety and efficacy of a topical gentamicin-collagen sponge in diabetic patients with a mild foot ulcer infection. SAGE Open Med 2018; 6:2050312118773950.

26. Lebowitz D, Gariani K, Kressmann B, et al. Are antibiotic-resistant pathogens more common in subsequent episodes of diabetic foot infection? Int J Infect Dis 2017; 59:61-64.

27. Reber A, Moldovan A, Dunkel N, et al. Should the methicillin-resistant Staphylococcus aureus carriage status be used as a guide to treatment for skin and soft tissue infections? J Infect 2012; 64:513-519.

28. Charles PG, Uçkay I, Kressmann B, et al. The role of anaerobes in diabetic foot infections. Anaerobe 2015; 34:8-13

29. Abbas M, Uçkay I, Lipsky BA. In diabetic foot infections antibiotics are to treat infection, not to heal wounds. Expert Opin Pharmacother 2015; $16: 821-832$.

30. Lipsky BA, Holroyd KJ, Zasloff M. Topical versus systemic antimicrobial therapy for treating mildly infected diabetic foot ulcers: a randomized, controlled, double-blinded, multicenter trial of pexiganan cream. Clin Infect Dis 2008; 47:1537-1545.

31. Chibber S, Kaur T, Kaur S. Co-therapy using lytic bacteriophage and linezolid: effective treatment in eliminating methicillin resistant Staphylococcus aureus (MRSA) from diabetic foot infections. PLoS One 2013; 8:56022.
32. Milne TE, Schoen DE, Bower VM, Gurr JM. Benchmarking healing times for diabetic foot ulcerations and investigating the influence of peripheral arterial disease and infection. J Foot Ankle Res 2011; 4:31.

33. Gariani K, Uçkay I, Lipsky BA. Managing diabetic foot infections: a review of the new guidelines. Acta Chir Belg 2014; 114:7-16.

34. Barwell ND, Devers MC, Kennon B, et al. Diabetic foot infection: antibiotic therapy and good practice recommendations. Int J Clin Pract 2017; 71.

35. Pence LM, Mock CM, Kays MB, et al. Correlation of adherence to the 2012 Infectious Diseases Society of America practice guidelines with patient outcomes in the treatment of diabetic foot infections in an outpatient parenteral antimicrobial programme. Diabet Med 2014; 31:1114-1120.

36. Catho $G$, De Kraker $M$, Waldispühl Suter B, et al. Study protocol for a multicentre, cluster randomised, superiority trial evaluating the impact of computerised decision support, audit and feedback on antibiotic use: the Computerized Antibiotic Stewardship Study (COMPASS). BMJ Open 2018; 8:022666.

37. Uçkay I, Vernaz-Hegi N, Harbarth $\mathrm{S}$, et al. Activity and impact on antibiotic use and costs of a dedicated infectious diseases consultant on a septic orthopaedic unit. J Infect 2009; 58:205-212.

38. Al-Mayahi M, Cian A, Lipsky BA, et al. Administration of antibiotic agents before intra-operative sampling in orthopedic infections alters culture results. J Infect 2015; 71:518-525.

39. Martínez-Gómez DA, Moreno-Carrillo MA, Campillo-Soto A, et al. Reduction in diabetic amputations over 15 years in a defined Spain population. Benefits of a critical pathway approach and multidisciplinary team work. Rev Esp Quimioter 2014; 27:170-179.

40. Nather A, Siok Bee C, Keng Lin W, et al. Value of team approach combined with clinical pathway for diabetic foot problems: a clinical evaluation. Diabet Foot Ankle 2010; 1:; Epub 2010 Dec 30

41. Sotto A, Richard JL, Combescure C, et al. Beneficial effects of implementing guidelines on microbiology and costs of infected diabetic foot ulcers. Diabetologia 2010; 53:2249-2255.

42. Lavigne JP, Sotto A, Dunyach-Remy C, Lipsky BA. New molecular techniques to study the skin microbiota of diabetic foot ulcers. Adv Wound Care (New Rochelle) 2015; 4:38-49.

43. Uçkay I, Ferry $T$, Stern R, et al. Use of serum antistreptolysin-O titers in the microbial diagnosis of orthopedic infections. Int J Infect Dis 2009; 13:421-424.

44. Uçkay I, Garzoni C, Ferry T, et al. Postoperative serum pro-calcitonin and Creactive protein levels in patients with orthopedic infections. Swiss Med Wkly 2010; 140:13124.

45. Lesens $\mathrm{O}$, Desbiez F, Vidal M, et al. Culture of per-wound bone specimens: a simplified approach for the medical management of diabetic foot osteomyelitis. Clin Microbiol Infect 2011; 17:285-291.

46. Kılıçoğlu Öl, Demirel M, Aktaş. New trends in the orthopaedic management of diabetic foot. EFORT Open Rev 2018; 3:269-277.

47. Kowalski TJ, Matsuda M, Sorenson MD, et al. The effect of residual osteomyelitis at the resection margin in patients with surgically treated diabetic foot infection. J Foot Ankle Surg 2011; 50:171-175.

48. Atway S, Nerone VS, Springer KD, Woodruff DM. Rate of residual osteomyelitis after partial foot amputation in diabetic patients: a standardized method for evaluating bone margins with intraoperative culture. J Foot Ankle Surg 2012; 51:749-752.

49. Mijuskovic $B$, Kuehl $R$, Widmer AF, et al. Culture of bone biopsy specimens overestimates rate of residual osteomyelitis after toe or forefoot amputation. J Bone Joint Surg Am 2018; 100:1448-1454.

50. Uçkay I, Jugun K, Gamulin A, et al. Chronic osteomyelitis. Curr Infect Dis Rep 2012; 14:566-575.

51. Bouvet C, Gjoni S, Zenelaj B, et al. Staphylococcus aureus soft tissue infection may increase the risk of subsequent staphylococcal soft tissue infections. Int J Infect Dis 2017; 60:44-48.

52. Lin SY, Lin NY, Huang YY, et al. Methicillin-resistant Staphylococcus aureus nasal carriage and infection among patients with diabetic foot ulcer. J Microbiol Immunol Infect 2018; pii: S1684-1182(18)30155-5.

53. Haleem A, Schultz JS, Heilmann KP, et al. Concordance of nasal and diabetic foot ulcer staphylococcal colonization. Diagn Microbiol Infect Dis 2014; 79:85-89. 